\title{
Axisymmetric thermo-mechanical analysis of laser-driven noncontact transfer printing
}

\author{
Huang, Yonggang, y-huang@northwestern.edu, Northwestern University
}

\begin{abstract}
An axisymmetric thermo-mechanical model is developed for laser-driven noncontact transfer printing, which involves laser-induced impulsive heating to initiate separation at the interface between a soft, elastomeric stamp, and hard micro/nanomaterials (i.e., inks) on its surface due to a large mismatch in coefficients of thermal expansion. The result is the active ejection of the inks from the stamp, to a spatially separated receiving substrate, thereby representing the printing step. The model gives analytically the temperature field, and also a scaling law for the energy release rate for delamination at the interface between the stamp and an ink in the form of a rigid plate. The normalized critical laser pulse time for interfacial delamination depends only on the normalized absorbed laser power and width of the ink structure and has been verified by experiments.
\end{abstract}

